\title{
Que devient l'Église Évangélique d'Allemagne?
}

In West Germany, more than 1.5 million people say that they have left the Evangelical Church over the past 10 years. What is happening with this Church? Are we witnessing the beginning of a collapse? Or is this more now slowing down?

First of all we must look at the facts against the back-ground of the German Aufklärung. We will then see that whereas church membership is pitched at a high level, church tradition attaches greater importance to the demands of a life oriented by Christian morals than to attending services. But this move away from the Church must also be linked to a process at work in many other organizations: members who can no longer identify with the organization distance themselves from it. Where work within the Church is concerned, this implies that greater importance be attached to dialogue between the grass roots and those at the top.

En automne 1984, le président du Conseil de l'Église Évangélique d'Allemagne (E.K.D.), E. Lohse, présenta les résultats d'un sondage effectué parmi les membres de cette Église. Un fait mentionné un peu en marge de ce rapport attira l'attention: plus de 1,5 million de personnes avaient déclaré avoir quitté l'Église Évangélique en République Fédérale au cours des dix dernières années.

En réalité, ce chiffre n'est pas nouveau. Quiconque prend connaissance des rapports statistiques publiés par l'E.K.D. peut sans difficulté obtenir ce chiffre par simple addition. Mais l'attention accordée à ce chiffre met en lumière la nette incertitude qui règne tant parmi les dirigeants d'Églises que parmi les fidèles et l'opinion publique dès que la question de l'avenir de l'Église vient à être posée. Il s'agit en fait de la question de la forme de l'Église en tant qu'Église populaire (Volkskirche). Ce type d'incertitude était d'ailleurs à la base de l'étude réalisée par le bureau de l'E.K.D. Et sa récente publication sous la direction de J. Hanselmann, H. Hild et E. Lohse n'est pas un simple hasard (Was wird aus der Kirche? [Que devient l'Église] Güterlöher Verlag).

Cette étude a été réalisée à la demande de l'Église Évangélique d'Allemagne, de l'Église Évangélique Luthérienne de Bavière et de l'Église Évangélique de Hesse et Nassau. Un échantillon représentatif des membres de ces Églises (1500 personnes de plus de 14 ans) fut retenu dix ans après que le premier sondage de ce type eut été réalisé et rendu public en 1974 sous le titre "Wie stabil ist die Kirche?"' (Qu'en est-il de la stabilité de l'Église?). À l'époque, il avait été largement discuté dans le milieu ecclésiastique. 
Il serait erroné de penser que cette étude puisse être considérée comme un rapport tendancieux émanant des officiels de l'Église. L'interprétation des résultats y est méthodologiquement très soignée. Les nombreuses comparaisons qui y sont faites avec d'autres organisations met en évidence l'intérêt pratique de l'étude, à savoir l'intention du groupe d'étude de l'E.K.D. de la faire prendre en considération dans le cadre des réflexions sur le travail futur dans l'Église. Un rapport tendancieux aurait d'ailleurs provoqué des réactions au sein même de l'Église car y coexistent des orientations divergentes à propos de l'avenir et chacune, bien sûr, cherche à se légitimer non seulement au travers de ses propres idées mais également à partir de données sociales empiriques.

Au départ de ce sondage, une grande partie des questions posées en 1974 furent réutilisées. Ceci rend possible des comparaisons longitudinales. Des tendances apparaissent donc, bien que les difficultés méthodologiques liées à un tel processus d'enquête ne doivent pas être ignorées. Les responsables de l'étude ont dès lors souligné l'importance actuelle de la perspective transversale.

Que devient l'Église? Le million et demi d'abandons de l'Église au cours des dix dernières années n'est-il que le début d'un effondrement? Ou bien ce mouvement de la fin des années 1970 est-il en perte de vitesse? Dans le climat général actuellement plus conservateur, une récession du nombre des membres de l'Église est-elle encore à prévoir?

Ce qui s'était manifesté au début des années 1970 se confirme dix ans plus tard: la plupart des membres de l'Église Évangélique affirme un très haut degré d'appartenance. Ceci n'exclut pas que des opinions critiques très nettes s'expriment en nombre considérable. Toutefois, la majorité des fidèles tient à son Église. Un tiers des personnes interrogées se déclare - en 1982 comme en 1972 - lié à l'Église; un second tiers se dit quelque peu lié et le dernier tiers à peine lié ou pas du tout lié. Bien que l'on puisse observer de légères fluctuations de ces valeurs, on peut dire que, globalement, la situation n'a pas subi de changement profond au cours de ces dix années. Le climat est devenu un peu plus bienveillant à l'égard de l'Église, moins critique et contestataire. Ceci n'est d'ailleurs guère surprenant puisque les sociologues estiment qu'en matière de religion on assiste à un processus de transformation à long terme en regard duquel une période de dix années compte à peine.

Cette constatation se confirme lorsque l'on demande aux interviewés quelles sont les raisons de leur appartenance à l'Église. En 1972, plus de la moitié d'entre eux se déclaraient membres "parce que je suis chrétien"'. Un pourcentage analogue fournit la même réponse en 1982. Plus frappant paraît être le fait qu'en 1982 à peine plus d'un quart des personnes interrogées justifie le fait d'être membre de l'Église par l'affirmation «parce que je suis religieux" (cet item n'était pas prévu en 1972). La compréhension liée à l'affirmation «parce que je suis chrétien» semble donc manifestement plus vaste et multiforme. Dans le langage quotidien, celui qui se déclare «religieux» est plutôt considéré comme "pieux». Se dire "chrétien», par contre, est connoté d'une preuve de participation responsable à la vie civile. Cette façon de voir les choses par les protestants se confirme par les réponses à la question: «quelle est la condition indispensable pour être Évangélique?». Le baptême et la confirmation sont évidemment cités en premier lieu. Mais cette réponse est immédiatement suivie par celle qui dit: «s'efforcer d'être un homme honnête et fiable» ou de «poursuivre sa propre conscience». 
La piété protestante s'avère ainsi capable de se passer d'Église. Qui veut être chrétien ne doit pas obligatoirement participer à la vie ecclésiale. La conduite morale de sa vie est bien plus importante.

Cette attitude indique que la pratique religieuse ne doit pas nécessairement être prise comme un indice de la vitalité d'une communauté ecclésiale populaire (Église de masse).

Des chrétiens étrangers visitant la République Fédérale d'Allemagne observent souvent avec étonnement combien la pratique religieuse y est faible par rapport au nombre des membres de l'Église. Et dans les discussions publiques en Allemagne, le problème des églises vides est aussi quelquefois mis en évidence. Les participants réguliers au culte ne sont évidemment pas les derniers à ressentir cette absence d'une communauté plus importante comme problématique. Il faut toutefois tenir compte de ce que, dans la tradition de l'Aufklärung allemande, un style de vie chrétienne est né qui place la participation au culte bien après l'orientation morale-chrétienne de la vie.

Dans cette perspective, il est plus important d'être chrétien dans la quotidienneté et moins important de participer à la vie de la communauté chrétienne. Celui qui participe à la vie de la communauté est considéré comme «pieux», ce qui est souvent associé dans le langage courant à une attitude «bigote». Il ne fait pas de doute que cette façon de voir demeure toujours prédominante parmi les membres de l'Église Évangélique, même si dans la théologie et dans l'Église, la participation au culte a reconquis de l'importance par rapport à ce qu'elle avait été dans la tradition de l'Aufklärung et du XIX ${ }^{\mathrm{e}}$ siècle bourgeois.

Quoiqu'il en soit, un tiers seulement des membres interrogés affirme ne jamais participer au culte. Un autre tiers y est présent au moins quelques fois par an, y compris les dimanches ordinaires. Le dernier tiers n'y participe qu'à l'occasion de fêtes ou d'événements familiaux. Mais il s'agit là de l'appréciation subjective de chacun à propos de sa propre pratique. La participation réelle aux offices est généralement inférieure à cette estimation. Enfin, en ce qui concerne la place accordée par chacun au culte, il faut savoir que la comparaison des taux de participation entre 1972 et 1982 révèle une légère progression au cours de ces années.

Selon l'opinion de ses membres, l'Église se réalise de façon particulièrement manifeste dans son engagement diaconal. L'amour du prochain au niveau individuel doit se traduire par la diaconie au niveau de l'Église institutionnelle. À cet égard, il y a peu de changement dans les opinions depuis dix ans. Toutefois, il apparaît que les membres de l'Église attendent d'elle qu'elle soit en mesure de faire face aux défis lancés actuellement dans ce domaine. Ainsi, par exemple, l'aide accordée par l'Église aux pays en développement est considérée comme plus importante qu'elle ne l'était en 1972. On considère aussi que les problèmes naissant du chômage devraient être plus sérieusement abordés qu'ils ne le sont actuellement. Et il est particulièrement instructif de constater que le soutien au mouvement pour la paix est considéré comme un domaine important d'activité de l'Église ( $72 \%$ des personnes interrogées pensent qu'il serait bon que l'Église soutienne ce mouvement). Ce résultat est frappant dans la mesure où la prise de positions publiques de l'Église face aux questions politiques actuelles n'est attendue que par un nombre plus restreint d'interviewés et qu'un nombre équivalent d'entre eux (27\%) estiment ces pri- 
ses de positions ni jutifiées ni importantes. Manifestement, le mot paix symbolise une valeur qui se situe au-delà des querelles des partis politiques et est considéré comme fondamental pour la vie humaine future.

C'est précisément à ce sujet que l'Église est mise en question. Elle est impliquée dans des problèmes fondamentaux qui sont à résoudre non seulement sur base de l'avis des théologiens mais aussi à partir de l'opinion des membres de l'Église.

Mais alors, pourquoi tant de personnes tournent-elles le dos à l'Église en la quittant? À côté de la majorité qui soutient l'Église, une nette minorité la critique et même la refuse. Et cette minorité se trouve mieux tolérée dans la vie publique: quelqu'un qui abandonne l'Église ne s'expose pas à des soupçons d'ordre moral mais, au contraire, manifeste un comportement jugé civilement normal. Ainsi, en 1982, par comparaison avec 1972, un nombre plus grand de membres ont déjà réfléchi à la démarche de l'abandon de l'Église. Cela signifie que celui qui a une attitude critique à l'égard de l'Église, celui qui «ne peut pas en faire grand chose», celui qui désapprouve la façon dont elle réalise la tradition chrétienne peut avoir plus que dans le passé tendance à mettre fin à son appartenance. Cela tient également au fait que ce n'est qu'en s'affirmant dans le présent que la tradition est acceptée et soutenue.

Tout ceci étant dit, on ne doit pas s'attendre à une diminution du nombre des abandons à l'égard de l'Église. Il ne s'agit pas d'un mouvement de fuite, mais d'un processus auquel bien d'autres organisations se trouvent confrontées: les membres qui ne peuvent s'identifier avec l'organisation prennent leurs distances.

Certes, il est toujours plus difficile de quitter l'Église qu'un syndicat. Cette démarche suppose pour chacun, même pour celui qui a pris distance, un effort de réflexion et de décision intérieure. Mais cette démarche est possible et est effectuée très consciemment par ceux qui tentent de voir clair en euxmêmes.

Tout ceci signifie pour les responsables et dirigeants des Églises ainsi que pour les communautés ecclésiales que, dans l'avenir, l'action menée devra se justifier et être rendue compréhensible pour les membres. Il y a là une invitation à entraîner les membres de l'Église dans une réflexion et à se laisser davantage déterminer par le fruit de cette réflexion. Cela ne veut pas dire que l'Église devrait se plier aux opinions majoritaires mais bien que le dialogue dans les Églises devrait acquérir de l'intensité. Des changements sont soutenus et même voulus par les membres de l'Église. Le maintien de la tradition est une tâche reconnue comme importante par beaucoup d'entre eux également. C'est la raison pour laquelle le dialogue offre de nouvelles chances d'avenir, même s'il peut mener aussi à des conflits. 